\title{
The CSCL field matures
}

\author{
Gerry Stahl • Friedrich Hesse
}

Published online: 9 January 2010

(C) International Society of the Learning Sciences, Inc.; Springer Science+Business Media, LLC 2009

\section{The Web of Science}

As it enters its fifth year of publication, $i j C S C L$ has learned that it has been selected for coverage in Thomson Reuters products and services (formerly ISI). Beginning with Volume 3, Number 1, 2008, ijCSCL will be indexed and abstracted in the Web of Science under the following categories:

- Social Sciences Citation Index ${ }^{\circledR} /$ Social Scisearch ${ }^{\circledR}$

- Journal Citation Reports/Social Sciences Edition

- Current Contents ${ }^{\circledR} /$ Social and Behavioral Sciences

Because the journal was accepted starting with 2008, the first Impact Factor will be calculated for 2010, and will be published in June 2011 .

This is the most prestigious form of indexing for academic journals. Universities and other institutions in many countries consider journals indexed by ISI to be top-rank publications in matters of tenure and promotion. It is rare for new journals to be accepted for indexing so quickly. ijCSCL has been considered the logical place to publish major contributions to the field of CSCL ever since it was founded by the CSCL community in 2006. However, now, the decision by ISI should mean that scholars working in the broader field will — even more than in the past — consider $i j C S C L$ to be a premier publication venue.

ISI's announcement is not only a tribute to the Editorial Board and many other reviewers who have worked hard to guide authors to meet high standards of academic publication. It is also due to the authors who took the risk to publish in a new journal and the readers who have subscribed through ISLS and supported the journal.

More than anything else, the journal's increased stature is a clear and direct reflection of the maturing of the field of CSCL. The history of the field can be traced to a workshop in

\footnotetext{
G. Stahl $(\bowtie)$

Drexel University, Philadelphia, PA, USA

e-mail: Gerry.Stahl@drexel.edu

F. Hesse

Knowledge Media Research Center, Tubingen, Germany

e-mail: f.hesse@iwm-kmrc.de
} 
Maratea, Italy, in 1989. The establishment of a regular biannual CSCL conference in 1995 defined a persistent research community. With the 2001 conference in Maastricht and the 2005 conference in Taipei, as well as the founding of ISLS as a supporting institution, the community became self-consciously international and permanent. The Springer CSCL book series and the Springer ijCSCL journal provide crucial publication outlets specifically founded for this field. The decision by ISI is a further landmark in the growth of our field.

\section{Volume 5, issue 1}

The institutional maturation of the CSCL field is matched by developments in the field's research and theory. This issue of the journal illustrates some of the changes.

This issue sees the publication of some substantial contributions - as partially reflected in their length. As Executive Editors, we are often asked how long a journal article should be, as though the goal was to produce a certain quantity of words. The answer is that the main consideration is to have something important to say, a significant contribution to the field. The length should be just enough to clearly express and support the claims - and no longer. A quick scan through the past four volumes shows that most articles averaged about twenty formatted journal pages. However, we are open to shorter articles: book reviews, notes, reports on international developments in CSCL. We are also open to occasional longer articles, as in this issue: The first paper presents a complex framework that requires lengthy motivation, presentation, and illustration and the second paper reviews, with impressive thoroughness, one of the most extensive realms of research in CSCL. Neither of these papers is likely to strike the interested reader as verbose; they are just long enough to convey their message.

The articles in this issue illustrate some ways in which the CSCL field is maturing. They demonstrate a continuing breadth of concern with theory, methodology, pedagogy, technology, sub-domains, and empirical investigation. At the same time, they show a heightened level of self-reflection and a greater depth of analysis. In particular, they illustrate an intense and ongoing effort within this diverse multidisciplinary field to understand how research elaborated within incommensurate theoretical frameworks can contribute productively to a field with concerns in common.

This year's opening article addresses the central problem of sequentiality in CSCL discourse: How are we to analyze, represent, and understand the ways in which one action takes up the contribution of a previous action in an online interaction? This temporal structure underlies the possibility of collaborative learning - of thought itself, whether individual or group - yet our theories and methods have not sufficiently focused on this fabric of interaction. Daniel D. Suthers, Nathan Dwyer, Richard Medina, and Ravi Vatrapu present the thinking of their lab in Hawaii over the past several years on this important theme.

One of the subareas of CSCL which has gotten perhaps the most attention is argumentation. A particularly clear way to look at collaborative learning is to study how people debate and argue about a claim. CSCL researchers have intensely explored a variety of technologies for computer support of argumentation, looking both at helping students to learn to argue effectively and at using argumentation skills to learn collaboratively. Oliver Scheuer, Frank Loll, Niels Pinkwart, and Bruce M. McLaren have joined efforts from their AI labs in Germany and the US to undertake a comprehensive review of this extensive and productive effort by the CSCL community. 
Structuring or scaffolding the sequential flow of student interactions has long been a central interest in CSCL, including supporting argumentation moves, scripting classroom discourse processes, or providing a selection of utterance categories (perhaps including labels, prompts, opening phrases). In their empirical analysis of the use of labeling under different conditions, Eva Mary Bures, Philip C. Abrami, and Richard F. Schmid of Canada argue that multiple forms of scaffolding may interfere with each other and that labeling should be designed flexibly so it can be tuned to the level of structure already existing in the educational activity.

On a theoretical and methodological level, the multidisciplinary field of CSCL has struggled with the substantial tensions, if not conflicts, between different approaches or even incommensurate paradigms in the work of different research groups. For instance, researchers in the cognitive science tradition favor quantitative studies aiming to measure the effects of mental representations of individuals, whereas researchers focused on situated interaction often opt for qualitative studies that reveal social practices, community participation, and group phenomena. There has been increasing discussion within the CSCL community about how to maintain a coherent and productive discourse with these diverse voices. Marc Clarà and Teresa Mauri of Spain close this issue of ijCSCL with the suggestion that multi-vocality in our field can be a healthy characteristic as long as we can find ways to bring the various findings into communication with each other. Focusing on content-analysis research, the authors identify three dimensions along which studies in this subfield of CSCL can be brought into dialectic relations with one another. 